\title{
Seismic migration of blended shot records with surface-related multiple scattering
}

\author{
D. J. (Eric) Verschuur ${ }^{1}$ and A. J. (Guus) Berkhout ${ }^{2}$
}

\begin{abstract}
This paper focuses on the concept of using blended data and multiple scattering directly in the migration process, meaning that the blended input data for the proposed migration algorithm includes blended surface-related multiples. It also means that both primary and multiple scattering contribute to the seismic image of the subsurface. Essential in our approach is that multiples are not included in the Green's functions but are part of the incident wavefields, utilizing the so-called double illumination property. We find that complex incident wavefields, such as blended primaries and/or blended multiples, require a reformulation of the imaging principle in order to provide broadband angle-dependent reflection properties.
\end{abstract}

\section{INTRODUCTION}

Multiple scattering is usually considered to be an undesired seismic phenomenon (multiples are qualified as noise). In the past, a variety of technologies were developed to remove multiple reflections from the data. This is particularly true for surface-related multiples. These multiples are generally very strong and, therefore, seriously mask the primary reflections. One of the most successful solutions is the family of surface-related multiple-elimination algorithms. These algorithms are based on wavefield prediction by multirecord convolution and on the wavefield operators being data-driven (Berkhout, 1982; Verschuur, 1991; Berkhout and Verschuur, 1997; Weglein et al., 1997; Dragoset, 1999). The same concept — prediction by multirecord convolution - has also been proposed for internal multiples (Berkhout, 1982). The problem of internal-multiple removal is more difficult to solve, but successful applications have been published by Weglein et al. (1997) and by Berkhout and Verschuur (2005).

After successful multiple removal, primary reflections remain and they function as input to the migration process. Many migration algorithms exist, based on ray- or beam-tracing (see e.g., Keho and Beydoun, 1988), Kirchhoff summation (Schneider, 1978), and oneway finite difference (Claerbout, 1976). A major challenge in migration algorithms is maintaining wide-angle (preferably beyond $90^{\circ}$ ) reflections in the wavefield extrapolation process. For this reason, we now observe that two-way, finite-difference extrapolation algorithms in a smooth background medium have gained a lot of interest. Application of reverse time migration (RTM) to shot records is based on the two-way extrapolation technology (Baysal et al., 1983; Biondi and Shan, 2002). Another major migration issue is the velocity model. Today, we see that the new generation of low-frequency, full-waveform velocity inversion algorithms opens new opportunities for better migration velocities (Virieux and Operto, 2009).

The quality of the (angle-dependent) seismic-migration output can be significantly improved if multiple scattering is utilized in the incident wavefield. Furthermore, by including the concept of blending (see, e.g., Beasley et al., 1998; Stefani et al., 2007; Berkhout, 2008), a dense and broadband illumination of the subsurface is obtained with a limited number of physical experiments. In this paper, we will demonstrate this property for the relatively strong surfacerelated multiples.

\section{FROM SINGLE TO DOUBLE ILLUMINATION}

There is increasing evidence that multiples contain a lot of valuable subsurface information. This means that multiples should not be removed and thrown away, but they should be used to improve seismic migration and inversion results. Several past publications show the use of multiples in the migration process. Reiter et al. (1991) extend the Kirchhoff migration operators to include waterlayer multiple ray paths. Applications of this concept were also found in the field of VSPs, in which primaries provide a very limited illumination area and multiples can extend the image space considerably (Jiang et al. 2005, 2007). Brown and Guitton (2005) propose a joint inversion method to find subsurface information based on primaries and multiples simultaneously. However, their implementation was geared toward a locally laterally invariant earth via the

\footnotetext{
Manuscript received by the Editor 7 May 2010; revised manuscript received 21 July 2010; published online 6 January 2011.

${ }_{1}^{1}$ Delft University of Technology, Faculty of Applied Sciences, Delft, the Netherlands. Email: d.j.verschuur@tudelft.nl.

${ }^{2}$ Delft University of Technology, Faculty of Civil Engineering and Geosciences, Delft, the Netherlands. Email: a.j.berkhout@ tudelft.nl. (C) 2011 Society of Exploration Geophysicists. All rights reserved.
} 
CMP domain, handling only preselected specular reflections. A more advanced implementation of imaging multiples can be obtained via shot-record migration (Berkhout, 1982; Berkhout and Verschuur, 1994; Youn and Zhou, 2001; Guitton, 2002). As we will also show, migration of multiples involves a more complex downgoing source wavefield. In the case of ocean-bottom cable data, the full downgoing wavefield and resulting upgoing wavefield can be extracted directly from the multicomponent measurements and fed into a shot-record migration scheme, as demonstrated by Muijs et al. (2007). However, another solution is required for recordings at the surface. We will follow the shot-record approach that we proposed in 1994 (Berkhout and Verschuur, 1994). New is the more advanced imaging condition, allowing the recovery of angle-dependent reflection as well. We will further show that this extended imaging condition also accommodates blended seismic data.

The role of multiples as a secondary illumination of the subsurface can be easily understood from the feedback model (Figure 1). In the following, we will use a formulation in the space-frequency domain (Berkhout, 1982):

$$
\begin{aligned}
& \mathbf{P}_{0}^{-}=\mathbf{X}_{0} \mathbf{S}^{+} \text {(primary data, single illumination), } \\
& \mathbf{P}^{-}=\mathbf{X} \mathbf{S}^{+} \text {(total data, single illumination), }
\end{aligned}
$$

or

$$
\mathbf{P}^{-}=\mathbf{X}_{0} \mathbf{Q}^{+} \text {(total data, double illumination), }
$$

where

$$
\mathbf{Q}^{+}=\mathbf{R}^{\cap} \mathbf{P}^{-}+\mathbf{S}^{+} \text {(total downgoing wavefield). }
$$

In equations $1 \mathrm{a}$ and $1 \mathrm{c}$, the subscript 0 represents the situation without surface-related multiples. In equations $1 \mathrm{~b}-1 \mathrm{~d}$, matrices $\mathbf{P}^{-}$and $\mathbf{Q}^{+}$represent the multidimensional upgoing and downgoing wavefields at the surface $\left(\mathrm{z}_{0}\right)$, respectively. Each column of matrix $\mathbf{S}^{+}$ represents a downgoing manmade source wavefield leaving the surface (geometry and signature). Transfer operator $\mathbf{X}_{0}$ quantifies wavefield propagation and reflection in the subsurface $\left(z>z_{0}\right)$ that may include any complexity such as refraction and diffraction. Operator $\mathbf{R}^{\cap}$ equals the reflectivity at the lower side of the surface ( $\mathbf{z}$ $=\mathrm{z}_{0}$ ). Note the difference between operators $\mathbf{X}$ and $\mathbf{X}_{0}$. Operator $\mathbf{X}$ includes the strong surface reflectivity, but in $\mathbf{X}_{0}$ the reflective surface is not present. It can be easily seen from equations $1 \mathrm{c}$ and $1 \mathrm{~d}$ that



Figure 1. Feedback model, showing the upgoing and downgoing wavefields at the surface. Note that the surface-related multiples are generated by feedback operator $\mathbf{R}^{\cap}$. Also note that all surface-reflected wavefields re-illuminate the subsurface, leading to the concept of double illumination. impulse response matrix $\mathbf{X}_{0}$ is not only present in the primaries $\left(\mathbf{P}_{0}^{-}\right.$ $\left.=\mathbf{X}_{0} \mathbf{S}^{+}\right)$, but is also present in the multiples $\left(\mathbf{M}^{-}=\mathbf{X}_{0} \mathbf{R}^{\cap} \mathbf{P}^{-}\right)$. Hence, both wavefields contain information about subsurface operator $\mathbf{X}_{0}$.

The difference between $\mathbf{X}$ and $\mathbf{X}_{0}$ is very important (Figure 1): $\mathbf{X}$ is a complex operator that contains complex Green's functions because it includes the highly reflective surface (represented by operator $\mathbf{R}^{\cap}$ ) and $\mathbf{X}_{0}$ is relatively simple because the influence of the reflective surface has been removed $\left(\mathbf{R}^{\cap}=0\right)$. The feedback model in Figure 1 relates the two (substitute equation $1 \mathrm{~d}$ in equation $1 \mathrm{c}$ ):

$$
\mathbf{P}^{-}=\mathbf{P}_{0}^{-}+\mathbf{X}_{0} \mathbf{R}^{\cap} \mathbf{P}^{-} \text {(primaries plus multiples), }
$$

$\mathbf{P}_{0}^{-}=\mathbf{X}_{0} \mathbf{S}^{+}$being the response of the earth with a reflection-free surface (representing primaries plus internal multiples) and $\mathbf{X}_{0} \mathbf{R}^{\cap} \mathbf{P}^{-}$ representing the surface-related multiples $\left(\mathbf{M}^{-}\right)$. Using equation 1a, equation $2 \mathrm{a}$ can also be written as

$$
\mathbf{P}^{-}=\mathbf{X}_{0}\left(\mathbf{S}^{+}+\mathbf{R}^{\cap} \mathbf{P}^{-}\right) \text {(double illumination), }
$$

showing double illumination, meaning that both primary sources $\mathbf{S}^{+}$ and secondary sources $\mathbf{R}^{\cap} \mathbf{P}^{-}$illuminate the subsurface.

Using equation $2 \mathrm{a}$, preprocessing algorithms are now being developed that separate primaries and multiples by simultaneously estimating $\mathbf{S}^{+}$and $\mathbf{X}_{0}$ from both the primary and multiple wavefields. Note the first proposals by Berkhout (2006), using the inverse data space, and by Van Groenestijn and Verschuur (2009), estimating a parameterized version of $\mathbf{X}_{0}$ directly from $\mathbf{P}^{-}$. Parameterization can be done with bandlimited spikes (Van Groenestijn and Verschuur, 2009), with curvelets (Lin and Herrmann, 2009), or with Green's functions (Berkhout and Verschuur, 2010). Note that with such parameterization methods, the effective downgoing source wavefield $\mathbf{S}^{+}$can be estimated (with its proper absolute amplitude scale) in order to build the total downgoing wavefield $\mathbf{Q}^{+}=\mathbf{R}^{\cap} \mathbf{P}^{-}+\mathbf{S}^{+}$. In fact, this information can be extracted because we make use of both primaries and multiples.

We envision that acquisition systems, measuring both pressure and particle velocity, will be routinely used to allow an accurate decomposition of upgoing and downgoing wavefields $-\mathbf{P}^{-}$and $\mathbf{R}^{\cap} \mathbf{P}^{-}$, respectively - without any assumption on $\mathbf{R}^{\cap}$. Early experiments in the marine environment show promising results (Cambois et al., 2009).

\section{MIGRATION OF COHERENT PRIMARIES AND MULTIPLES}

The double illumination concept shows that surface-related multiple removal can be omitted in the seismic migration scheme (compare equations $1 \mathrm{a}$ and $1 \mathrm{c}$ ). This can be done if we use total downgoing wavefield $\mathbf{Q}^{+}$instead of primary source wavefield $\mathbf{S}^{+}$in the forward extrapolation process of a shot-record migration algorithm. Figure 2 visualizes the computational diagram. It is important to realize that for complex incident wavefields, simple correlation - as used in the standard imaging principle - must be replaced by multidimensional wavefield deconvolution (Muijs et al., 2007) or, even better, by a least-squares minimization process (Verschuur and Berkhout, 2009). In terms of our matrix formulation,

$$
\left\|\mathbf{W}^{H} \mathbf{P}^{-}-\mathbf{R}^{\cup} \mathbf{W} \mathbf{Q}^{+}\right\|^{2}+\varepsilon^{2}\left\|\mathbf{R}^{\cup}\right\|^{2}=\text { minimum, }
$$


where $\mathbf{W}$ is the propagation operator, $\mathbf{W}^{\mathrm{H}}$ is the adjoint version, \|\|$^{2}$ denotes the $\mathrm{L} 2$ norm, and $\mathbf{R}^{\cup}$ is the unknown reflectivity. The minimum norm stabilization term in this equation, with weight factor $\varepsilon^{2}$, can optionally be replaced by other constraints, like an L1 norm or a lateral continuity constraint. Note that the forward and backward propagation processes are explained here in terms of applying the propagation operators $\mathbf{W}$ and $\mathbf{W}^{\mathrm{H}}$, respectively, but it may also be formulated by applying forward and reverse time modeling. Once the reflectivity operators are obtained from this inversion process, the angle-dependent reflection information can be easily extracted by transforming each column of the $\mathbf{R}$-matrix to the wavenumber or linear Radon domain (see Berkhout, 1982; de Bruin et al., 1990; Berkhout, 1997).

The message of equation 3 is not that the wavefield extrapolation processes are modified, but that the input and the output are changed. The source matrix $\mathbf{S}^{+}$is replaced by $\mathbf{Q}^{+}$and the output data matrix $\mathbf{P}_{0}^{-}$is replaced by $\mathbf{P}^{-}$, respectively. In addition, the well-known imaging principle (crosscorrelation) is replaced by a least-squares minimization process to avoid cross-talk in the migration output.

It is important to realize that migration of multiples $\left(\mathbf{M}^{-}\right)$is independent of the source signal: the result is always zero phase. This creates the possibility to estimate during migration the source signal at each depth level as well. This means that equation 3 is applied in three steps. First, we replace $\mathbf{P}^{-}$by $\mathbf{M}^{-}$(output of SRME) in equation 3 and use $\mathbf{Q}^{+}=\mathbf{R}^{\cap} \mathbf{P}^{-}$, yielding a first estimate of $\mathbf{R}^{\cup}$ (columns of matrix $\mathbf{R}^{\cup}$ ). This estimate of $\mathbf{R}^{\cup}$ is based on multiples only, meaning that it is independent of $\mathbf{S}^{+}$. Next, we replace $\mathbf{P}^{-}$ by $\mathbf{P}_{0}^{-}$and we use $\mathbf{Q}^{+}=\mathbf{S}^{+}$to solve for the unknown source signals (columns of matrix $\mathbf{S}^{+}$). Fi- nally, we take $\mathbf{Q}^{+}=\mathbf{S}^{+}+\mathbf{R}^{\cap} \mathbf{P}^{-}$to improve the estimate of $\mathbf{R}^{\cup}$ by using both primaries and multiples $\left(\mathbf{P}^{-}\right)$.

Figure 3 illustrates the principle on a single reflector at $200 \mathrm{~m}$ depth in a homogeneous velocity medium for a coarse selection of shot records. The top row shows one of the involved shot records and the bottom row shows the angle-dependent properties as extracted from the input data, displayed in the horizontal slowness domain. Note that the reflector has been chosen to have angle-independent reflection properties, such that we expect a constant amplitude reflection function within the minimum and maximum slowness values present in the data. Equation 3 was solved for using only five shot records, with a spatial sampling of $400 \mathrm{~m}$ between sources. The inversion was implemented such that the reflection operators were es-

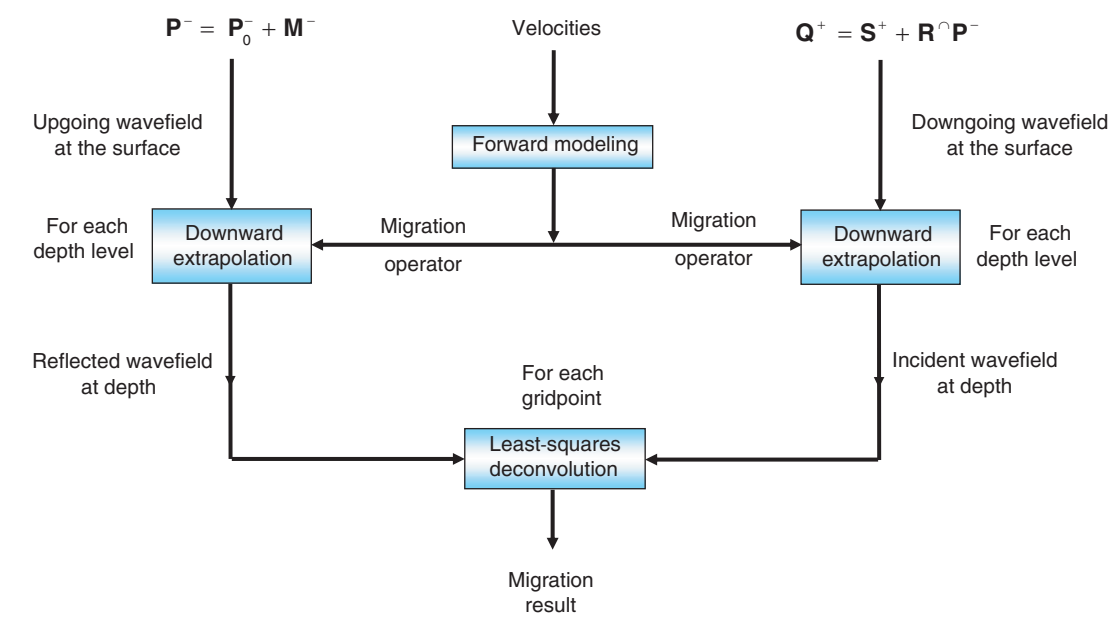

Figure 2. Using the double illumination concept (Figure 1), primaries $\left(\mathbf{P}_{0}^{-}\right)$and multiples $\left(\mathbf{M}^{-}\right)$are simultaneously migrated. Hence, for migration purposes multiple removal is not required anymore and, above all, the rich information contained in multiples is utilized (Berkhout, 2009).

a)

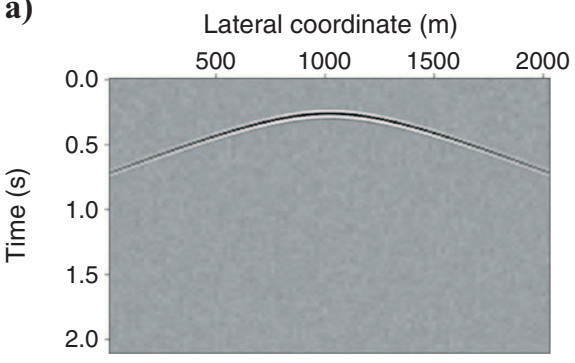

Lateral coordinate $(\mathrm{m})$

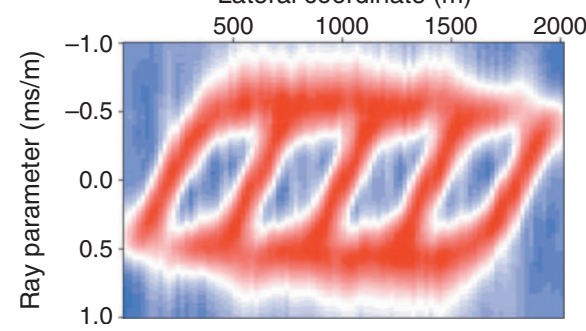

b)

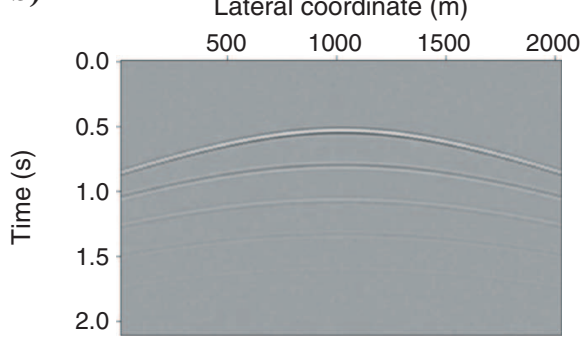

Lateral coordinate $(\mathrm{m})$

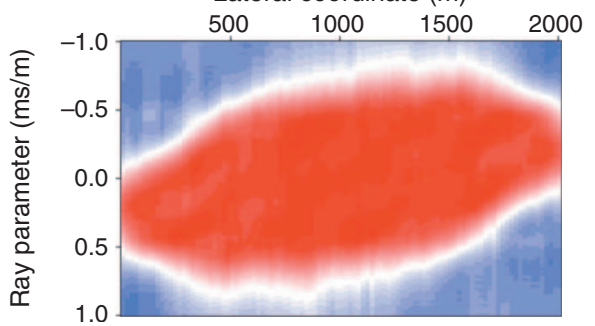

c)
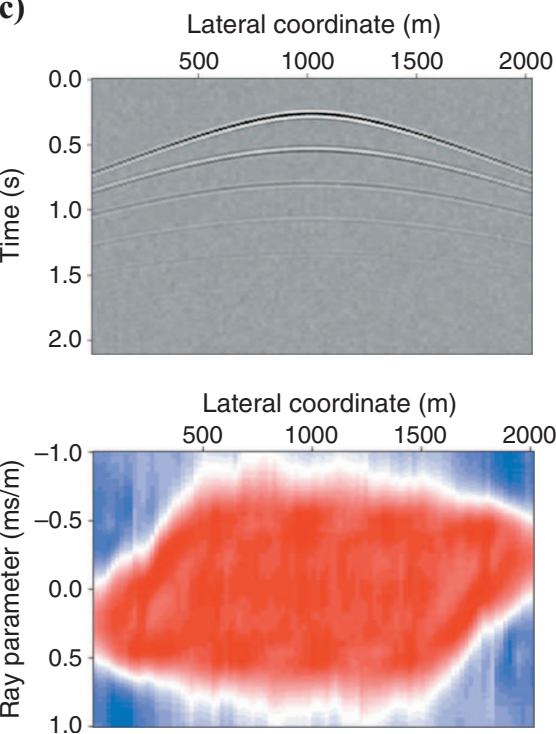

Figure 3. Angle-dependent migration result for five selected shot records ( $\Delta \mathrm{x}=400 \mathrm{~m}$ ) using (a) primaries, (b) multiples, and (c) primaries plus multiples. The top row shows one of the five shots and the bottom row displays the result of angle-dependent imaging along the boundary at $200 \mathrm{~m}$ depth. It is interesting to observe how well multiples illuminate the reflector. This property confirms our expectation that multiples are going to play an important role in future imaging technology. 
timated in the space-time domain as convolution operators that are short in time and space. Thus, this multidimensional imaging condition requires the solution of a small least-squares problem for each grid point in the subsurface. In Figure $3 \mathrm{a}$, one can observe that the obtained angle-dependent reflection information from the primaries-only data is very limited (almost single-fold illumination per reflector point). Note the excellent illumination properties of the multiples in Figure 3b. Note also that in Figure 3c primary and multiples were not separated, but were simultaneously migrated. For these examples, we estimated the source wavelet with the proper amplitude scale in order to ensure a well-balanced contribution from primaries and multiples in Figure 3c. It can be concluded that including multiples will greatly enlarge the subsurface illumination, being of great

importance for the 3D seismic situation where crossline source spacing in the order of $400 \mathrm{~m}$ is common.

\section{MIGRATION OF INCOHERENT PRIMARIES AND MULTIPLES}

In blended acquisition, time-overlapping shot records are generated in the field by using incoherent source arrays (Beasley et al., 1998; Stefani et al., 2007; Berkhout, 2008). It allows finer source sampling in the field in an attractive economic manner. It also allows accurate migration of rich 3D data sets (e.g., by wave equation migration or RTM), using fine source sampling with a small number of shot records. Figure 4 shows the computational diagram. In our algorithm, the reflectivity for each depth level is computed by an extension of equation 3 :



Figure 4. Simultaneous migration of blended primaries $\left(\mathbf{P}_{0}^{-} \Gamma\right)$ and blended multiples $\left(\mathbf{M}^{-} \Gamma\right)$. Note that wavefield deblending, signature deconvolution, and multiple removal are an integral part of the migration process (Berkhout, 2009).

$$
\begin{aligned}
& \left\|\mathbf{W}^{H} \mathbf{P}^{-} \Gamma-\mathbf{R}^{\cup} \mathbf{W} \mathbf{Q}^{+} \Gamma\right\|^{2}+\varepsilon^{2}\left\|\mathbf{R}^{\cup}\right\|^{2} \\
& \quad=\text { minimum, }
\end{aligned}
$$

where $\Gamma$ equals the blending operator. Berkhout (2008) proposes using simple time delays for the blending process. This type of blending allows the use of conventional sources in acquisition and, therefore, will be used in the next examples. Note that incident wavefields $\mathbf{Q}^{+} \boldsymbol{\Gamma}$ have superior illumination properties with respect to what is used today $\left(\mathbf{S}^{+}\right.$only), which is incoherent double illumination. The concept of using blended source wavefields in an (inversion-type) migration process was already demonstrated by Romero et al. (2000), Dai and Schuster (2009), and Tang and Biondi (2009), however, with the restriction of angle-independent scattering.

We repeat the example of Figure 3 by including blending as well. Figure 5a shows the blended a)

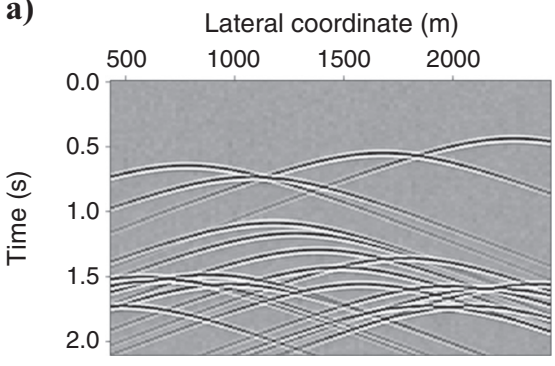

Lateral coordinate $(\mathrm{m})$

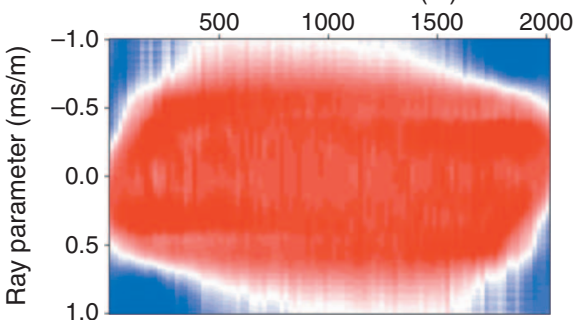

b)

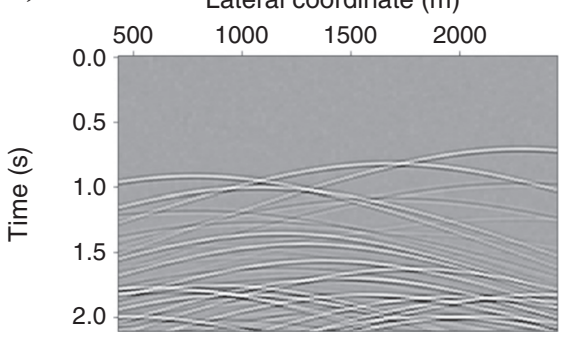

Lateral coordinate $(\mathrm{m})$

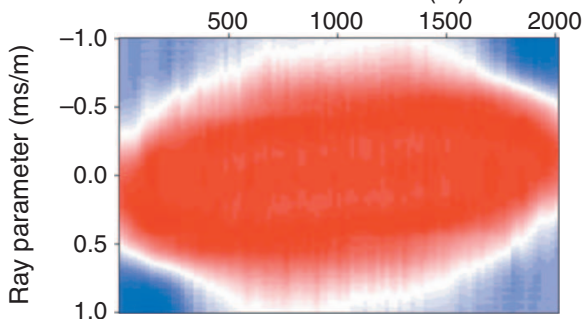

c)



Lateral coordinate $(\mathrm{m})$

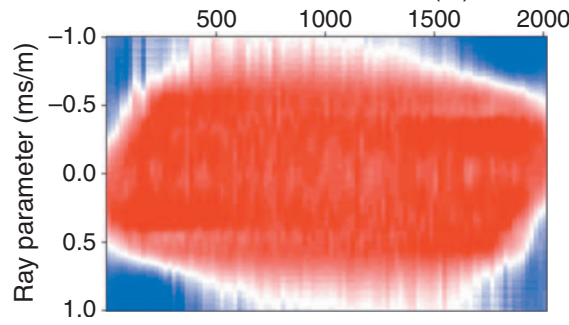

Figure 5. Angle-dependent migration result for five shot records with (a) blended primaries, (b) blended multiples, and (c) blended primaries plus blended multiples. The top row shows one of the five blended records and the bottom row shows the corresponding angle-dependent images at the reflector. The comparison with Figure 3 indicates that the use of multiples and the application of blending improve the quality of seismic images significantly. 
primary shot records and the angle-dependent migration result. The difference with Figure $3 \mathrm{a}$ is very clear: blending has significantly improved the illumination property. This conclusion can also be drawn by looking at the migrated blended multiples (compare Figure $5 \mathrm{~b}$ with $3 \mathrm{~b}$ ). Finally, the result of migrating the total blended shot record (blended primaries plus blended multiples) is shown in Figure $5 \mathrm{c}$. Note again the ex-cellent illumination property. Based on the

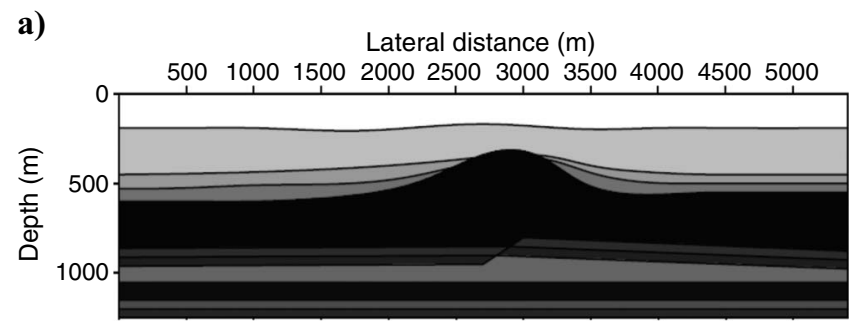

b)

Lateral coordinate $(\mathrm{m})$

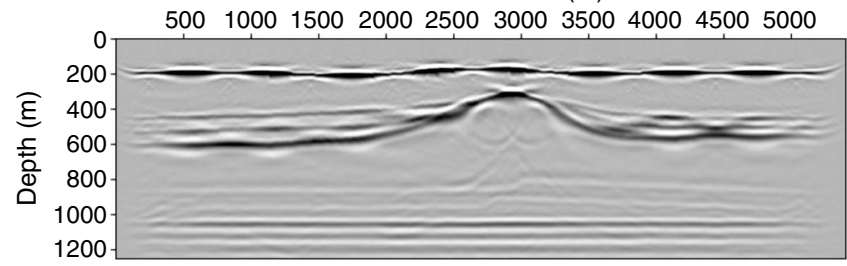

c)

Lateral coordinate $(\mathrm{m})$

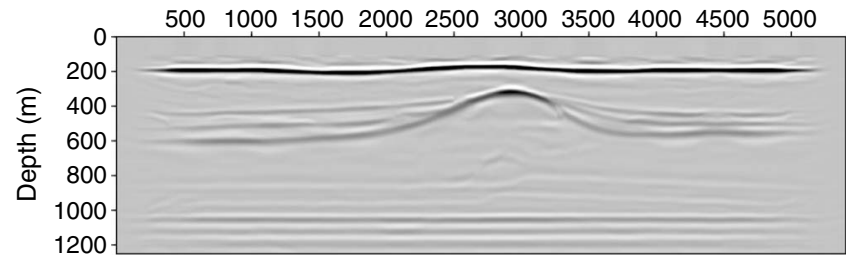

d)

Lateral coordinate $(\mathrm{m})$

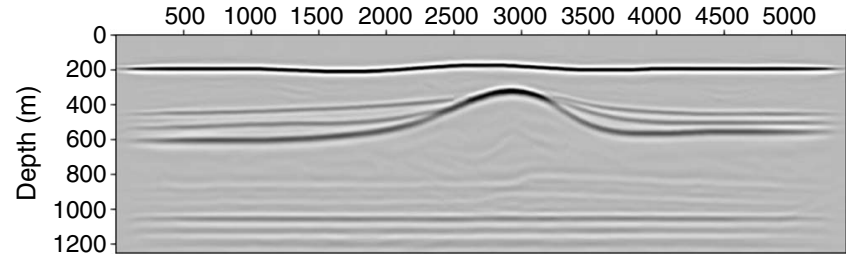

e)

Lateral coordinate $(\mathrm{m})$

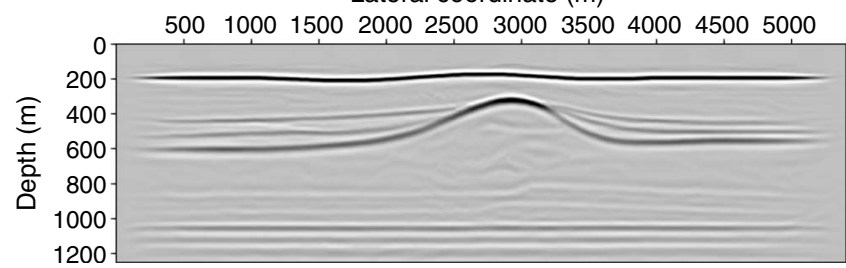

Figure 6. (a) Subsurface model with high velocity salt layer. (b) Prestack depth migration (PSDM) of nine primary shot records. (c) PSDM including multiples. (d) PSDM of nine primary blended shot records. (e) PSDM of blended primaries and multiples. results for Figures 3 and 5, we can conclude that either blending or including multiples provides a large improvement in illumination of coarsely sampled data.

Now, let us consider a more complex example, using the subsurface model of Figure 6a and finite-difference modeled data. We start with using nine unblended shot records, the sources having a separation of $600 \mathrm{~m}$. Note that this can represent the situation of coarse azimuth sampling. Figure $6 \mathrm{~b}$ shows the result of migrating primaries $\left(\mathbf{P}_{0}^{-}\right)$only. The depth image shows the estimated angle-averaged reflection coefficient for each subsurface point. The effect of coarse azimuth sampling is clearly visible. Next, we remigrate the same data but we now only use the surface multiples $\left(\mathbf{M}^{-}\right)$as input. The result is shown in Figure 6c. Note the significant improvement in the image.

Then, in another experiment, we use nine blended shot records, each incoherent source array consisting of 10 sources. Figure $6 \mathrm{~d}$ and e shows the migration result of the blended primaries and the blended primaries plus multiples, respectively. The difference with Figure $6 \mathrm{~b}$ (unblended primaries only) is significant. For the same number of shot records, the application of blending together with the utilization of surface multiples promise a major step forward in imaging.

Finally, we choose one boundary in the model (the top of the high velocity layer) and apply the angle-dependent imaging for the case of all primary shot records (Figure 7a, being the reference result), for nine shot records without and with multiples (Figure $7 \mathrm{~b}$ and c), and for nine blended shot records without and with multiples (Figure 7d and e). Note the poor illumination of using only nine primary shot records (compare Figure 7b with 7a). It is remarkable to observe the improved illumination and accurate reconstruction of angle-dependent reflection properties when including blending and/or multiples (Figure 7c-e), considering we are using only nine shot records.

\section{DISCUSSION}

As we have demonstrated, the use of blending and surface-related multiples will increase the illumination of the subsurface to a large extent. This works very well because the surface is a strong and deterministic reflection. Note that in our 2D examples (Figures 5-7), the effect of including multiples in blended data does not provide much extra illumination, because blending already contributed to a good subsurface coverage. However, in the full 3D case, blending and including multiples will have complementary effects in filling the offset-azimuth domain.

This concept could be extended to internal multiples, such that we can make use of indirect illumination of subsurface structures. Malcolm et al. (2009) demonstrate this property for the deterministic case of one known horizontal reflector that was used to image a complex area from below. Also, in the field of nondestructive testing, such as inspection of welds in pipelines, this is a known technique where the back wall is used for secondary illumination (see, e.g., Pörtzgen et al., 2007). 
a)

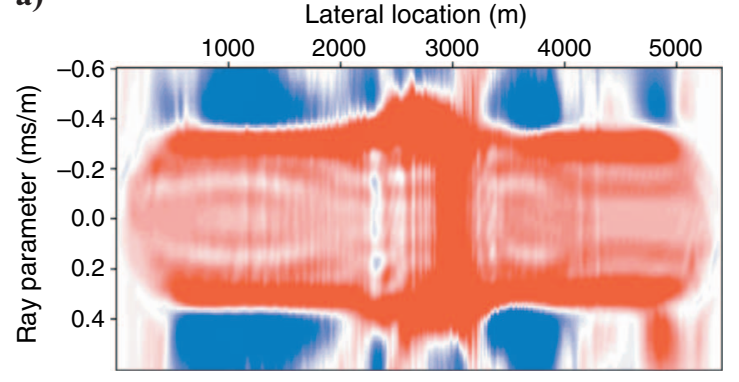

b)

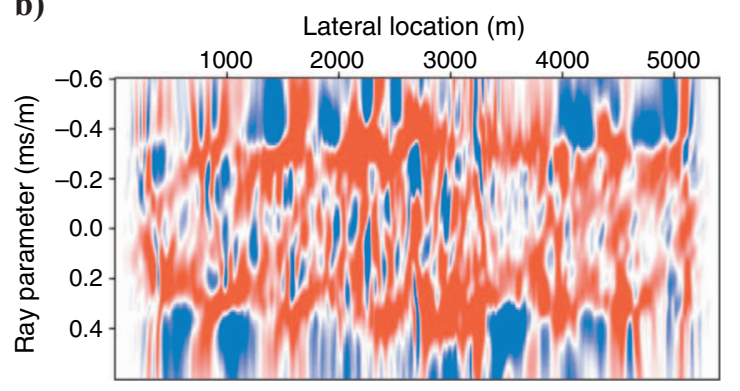

c)

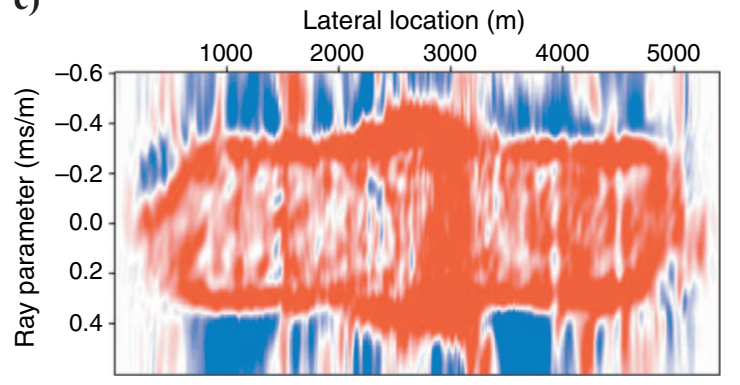

d)

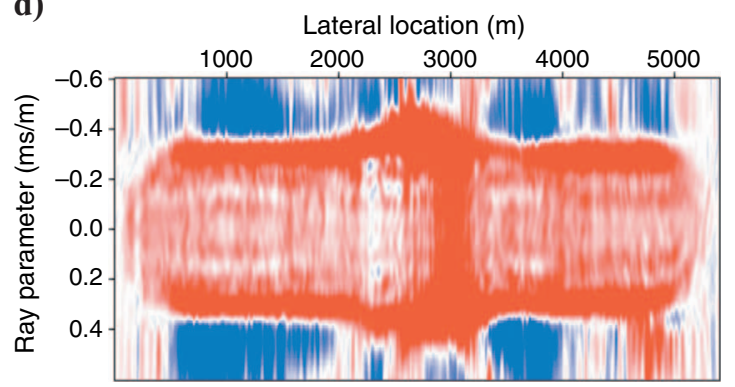

e)

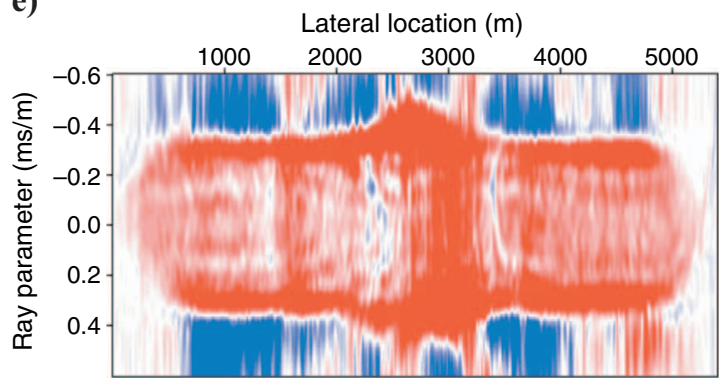

Figure 7. Angle-dependent image along the top of the high velocity layer in Figure 6a. (a) Reference result, using all 90 shot records, primaries only. (b) Result for nine primary shot records. (c) Result for nine shot records including multiples. (d) Result for nine blended primary shot records. (e) Result for nine blended shots with multiples.

\section{CONCLUSIONS}

Migration of blended primary wavefields can be generalized by including blended multiple scattering in the input data. In this paper, we focused on the relatively strong (blended) surface-related multiples.

In our solution, this generalization is not done by extending the Green's functions but by extending the incident wavefields, leading to the concept of double illumination. The advantage of this approach is that Green's functions stay the same and, therefore, no extra information of the subsurface is required.

Because of the complexity of the incident wavefields, a reformulation of the imaging principle, from wavefield correlation to wavefield minimization, is required. The involved inversion process can be efficiently implemented via the estimation of a short convolution filter for each subsurface grid point.

If primaries and multiples are simultaneously migrated, the separation of primaries and multiples (or the removal of multiples) is not needed anymore. Instead, knowledge of the source signature is required.

\section{REFERENCES}

Baysal, E., D. D. Kosloff, and J. W. C. Sherwood, 1983, Reverse time migration: Geophysics, 48, 1514-1524, doi: 10.1190/1.1441434.

Beasley, C. J., R. E. Chambers, and Z. Jiang, 1998, A new look at simultaneous sources: 68th Annual International Meeting, SEG, Expanded Abstracts, $133-135$

Berkhout, A. J., 1982, Seismic migration, imaging of acoustic energy by wavefield extrapolation - A. Theoretical aspects: Elsevier (second edition).

Berkhout, A. J., 2006, Seismic processing in the inverse data space: Geophysics, 71, no. 4, A29-A33, doi: 10.1190/1.2217727.

Berkhout, A. J., 2008, Changing the mindset in seismic data acquisition: The Leading Edge, 27, no. 7, 924-938, doi: 10.1190/1.2954035.

Berkhout, A. J., 2009, Grand challenges for geophysics, a seismic vision of the future: 79th Annual International Meeting, SEG, Expanded Abstracts, $3416-3420$.

Berkhout, A. J., and D. J. Verschuur, 1994, Multiple technology, part 2: Migration of multiple reflections: 64th Annual International Meeting, SEG, Expanded Abstracts, 1497-1500.

Berkhout, A. J., and D. J. Verschuur, 1997, Estimation of multiple scattering by iterative inversion, part I: Theoretical considerations: Geophysics, 62, 1586-1595, doi: 10.1190/1.1444261.

Berkhout, A. J., 1997, Pushing the limits of seismic imaging, part II: integration of prestack migration, velocity estimation, and AVO analysis: Geophysics, 62, 954-969, doi: 10.1190/1.1444202.

Berkhout, A. J., and D. J. Verschuur, 2005, Removal of internal multiples with the common focus-point (CFP) approach: Part 1 - Explanation of the theory: Geophysics, 70, no. 3, V45-V60, doi: 10.1190/1.1925753.

Berkhout, A. J., and D. J. Verschuur, 2010, Parameterization of seismic measurements by FPRs - Combining physics with mathematics: 72nd Annual International Meeting, EAGE, Extended Abstracts, G039.

Biondi, B., and G. Shan, 2002, Prestack imaging of overturned reflections by reverse time migration: 72nd Annual International Meeting, SEG, Expanded Abstracts, 1284-1287.

Brown, M. P., and A. Guitton, 2005, Least-squares joint imaging of multiples and primaries: Geophysics, 70, no. 5, S79-S89, doi: 10.1190/1.2052471.

Cambois, G., D. Carlson, C. Jones, M. Lesnes, W. Söllner, and H. Tabti, 2009, Dual sensor streamer data: Calibration, acquisition QC and attenuation of seismic interferences and other noises: 79th Annual International Meeting, SEG, Expanded Abstracts, 142-146.

Claerbout, J. F., 1976, Fundamentals of geophysical data processing: McGraw-Hill.

Dai, W., and G. T. Schuster, 2009, Least-squares migration of simultaneous sources data with a deblurring filter: 79th Annual International Meeting, SEG, Expanded Abstracts, 2990-2994.

de Bruin, C. G. M., C. P. A. Wapenaar, and A. J. Berkhout, 1990, Angle-dependent reflectivity by means of prestack migration: Geophysics, 55, 1223-1234, doi: 10.1190/1.1442938.

Dragoset, W. H., 1999, A practical approach to surface multiple attenuation: The Leading Edge, 18, no. 1, 104-108, doi: 10.1190/1.1438132.

Guitton, A., 2002, Shot-profile migration of multiple reflections: 72nd Annual International Meeting, SEG, Expanded Abstracts, 1296-1299. 
Jiang, Z., J. Sheng, J. Yu, G. T. Schuster, and B. E. Hornby, 2007, Migration methods for imaging different-order multiples: Geophysical Prospecting, 55, no. 1, 1-19, doi: 10.1111/j.1365-2478.2006.00598.x.

Jiang, Z., J. Yu, G. T. Schuster, and B. E. Hornby, 2005, Migration of multiples: The Leading Edge, 24, no. 3, 315-318, doi: 10.1190/1.1895318.

Keho, T. H., and W. B. Beydoun, 1988, Paraxial ray Kirchhoff migration: Geophysics, 53, 1540-1546, doi: 10.1190/1.1442435.

Lin, T. Y., and F. J. Herrmann, 2009, Unified compressive sensing framework for simultaneous acquisition with primary estimation: 79th Annual International Meeting, SEG, Expanded Abstracts, 3113-3117.

Malcolm, A., B. Ursin, and M. de Hoop, 2009, Seismic imaging and illumination with internal multiples: Geophysical Journal International, 176, no. 3, 847-864, doi: 10.1111/j.1365-246X.2008.03992.x.

Muijs, R., J. O. A. Robertsson, and K. Holliger, 2007, Prestack depth migration of primary and surface-related multiple reflections: Part I — Imaging: Geophysics, 72, no. 2, S59-S69, doi: 10.1190/1.2422796.

Pörtzgen, N., A. Gisolf, and G. Blacquière, 2007, Inverse wave field extrapolation: A different NDI approach to imaging defects: IEEE Transactions on UFFC, 54, 118-126.

Reiter, E. C., M. N. Toksöz, T. H. Keho, and G. M. Purdy, 1991, Imaging with deep-water multiples: Geophysics, 56, 1081-1086, doi: 10.1190/ 1.1443119.

Romero, L. A., D. C. Ghiglia, C. C. Ober, and S. A. Morton, 2000, Phase encoding of shot records in prestack migration: Geophysics, 65, no. 2, 426-436, doi: 10.1190/1.1444737.
Schneider, W. A., 1978, Integral formulation for migration in two and three dimensions: Geophysics, 43, 49-76, doi: 10.1190/1.1440828.

Stefani, J., G. Hampson, and E. F. Herkenhoff, 2007, Acquisition using simultaneous sources: 69th Annual International Meeting, EAGE, Extended Abstracts, B006.

Tang, Y., and B. Biondi, 2009, Least-squares migration/inversion of blended data: 79th Annual International Meeting, SEG, Expanded Abstracts, 2859-2863.

van Groenestijn, G. J. A., and D. J. Verschuur, 2009, Estimating primaries by sparse inversion and application to near-offset data reconstruction: Geophysics, 74, no. 3, A23-A28, doi: 10.1190/1.3111115.

Verschuur, D. J., 1991, Surface-related multiple elimination: An inversion approach: Ph.D. thesis, Delft University of Technology, Delft, the Netherlands.

Verschuur, D. J., and A. J. Berkhout, 2009, Target-oriented, least-squares imaging of blended data: 79th Annual International Meeting, SEG, Expanded Abstracts, 2889-2893.

Virieux, J., and S. Operto, 2009, An overview of full-waveform inversion in exploration geophysics: Geophysics, 74, no. 6, WCC1-WCC26, doi: 10.1190/1.3238367

Weglein, A. B., F. A. Gasparotto, P. M. Carvalho, and R. H. Stolt, 1997, An inverse scattering series method for attenuating multiples in seismic reflection data: Geophysics, 62, 1975-1989, doi: 10.1190/1.1444298.

Youn, O. K., and H. W. Zhou, 2001, Depth imaging with multiples: Geophysics, 66, 246-255, doi: 10.1190/1.1444901 